\title{
Evaluation of Flying Ad Hoc Network Topologies, Mobility Models, and IEEE Standards for Different Video Applications
}

\author{
Ghassan A. QasMarrogy \\ Department of Communication and Computer Engineering, College of Engineering, Cihan University-Erbil, \\ Kurdistan Region, Iraq
}

\begin{abstract}
Nowadays, drones became very popular with the enhancement of the technological progress of moving devices with a connection to each other, known as Flying Ad Hoc Network (FANET). It is used in most worldwide necessary life scenarios such as video recording, search and rescue, military missions, moving items between different areas, and many more. This leads to the necessity to evaluate different network strategies between these flying drones, which are essential to improve their quality of performance in the field. Several challenges must be addressed to effectively use FANET, to provide stable and reliable transmission for different types of data during vast changing topologies, such as different video sizes, different types of mobility models, recent Wireless Fidelity standards, types of routing protocols used, security problems, and many more. In this paper, a fully comprehensive analysis of FANET will be done to evaluate and enhance these challenges that concern different video types, mobility models, and IEEE 802.11n standards for best performance, by measuring throughput, retransmission attempt, and delay metrics. The result shows that Gauss-Markov mobility model gives the highest result using Ad Hoc On-Demand Vector and lowest delay, whereas for retransmission attempts, $2.4 \mathrm{GHz}$ frequency has the lowest as it can reach more coverage area than $5 \mathrm{GHz}$.
\end{abstract}

Index Terms-Video transmission; flying ad hoc network; mobility model; 2.4-5 GHz standards; routing protocols

\section{INTRODUCTION}

Recently, mobile networks became very popular with the advance in the technology sector, as they can move and fly at different speeds, equipped with multitools to offer different types of application services such as video recording, item carrying, search and rescue, military operations, and many more. There are different types of these mobile networks, namely, ad hoc network, mobile ad hoc network (MANET),

\section{ARO-The Scientific Journal of Koya University}

Vol. IX, No.1 (2021), Article ID: ARO.10764, 82 pages

DOI:10.14500/aro.10764

Received: 07 December 2020; Accepted: 14 February 2021

Regular research paper: Published: 08 May 2021

Corresponding author's e-mail: ghassan.qasmarrogy@

cihanuniversity.edu.iq

Copyright (C) 2021 Ghassan A. QasMarrogy. This is an open-access article distributed under the Creative Commons Attribution License. vehicular ad hoc networks, and flying ad hoc network (FANET) (Kaur and Singh, 2018), all of them use different types of routing protocols to communicate as they change their topology frequently. FANET is a set of moving drones that change its topology consistently without any fixed infrastructure while communicating with a base station from different heights, as shown in Fig. 1 (Marrogy, 2020).

One of the main requirements of FANET is video recording, it is used to capture high-quality videos while flying at different speeds across the area. The formats of the recorded video play a key role in data transmission between the drones, as it needs more time and higher bandwidth B.W to transfer high-quality videos during movement, this also requires better converging between the routing protocols to route the big size packets from node to node (Zheng, Sangaiah and Wang, 2018). Therefore, due to the fast movement of FANET causing the topology to be changed rapidly, it is difficult to capture and transmit high-quality videos between the nodes while movement, which gives FANET limited capability in transmitting. Furthermore, different other challenges and constraints are fronting FANET such as drone's scalability, limited B.W, different types of data transmission, different types of routing protocols, and the time required to finish tasks (Khan, Safi, Qureshi and Khan, 2017).

To communicate between drones, an IEEE 802.11 wireless adapter is needed to transmit with different frequencies such as $2.4 \mathrm{GHz}, 5 \mathrm{GHz}$, and $6 \mathrm{GHz}$ frequency bands. A numbering scheme was used by the Wireless Fidelity (WiFi) Alliance as a WIFI generation 802.11b, 802.11a, 802.11g, $802.11 \mathrm{n}, 802.11 \mathrm{ac}$, and 802.11ax protocols, respectively (Deng, et al., 2020), whereas some of these generations can support a dual band of $2.4 \mathrm{GHz}$ and $5 \mathrm{GHz}$ together (Karmakar, Chattopadhyay and Chakraborty, 2017). Table I shows the list of WIFI 802.11n standards.

Due to the mentioned challenges, an analysis is needed to test and simulate different types of realistic scenarios for FANET, to determine the best results and parameters for video data transmission.

In this paper, different types of realistic scenarios will be evaluated and analyzed for FANET, to simulate and find the best results for the mentioned challenges, concerning 


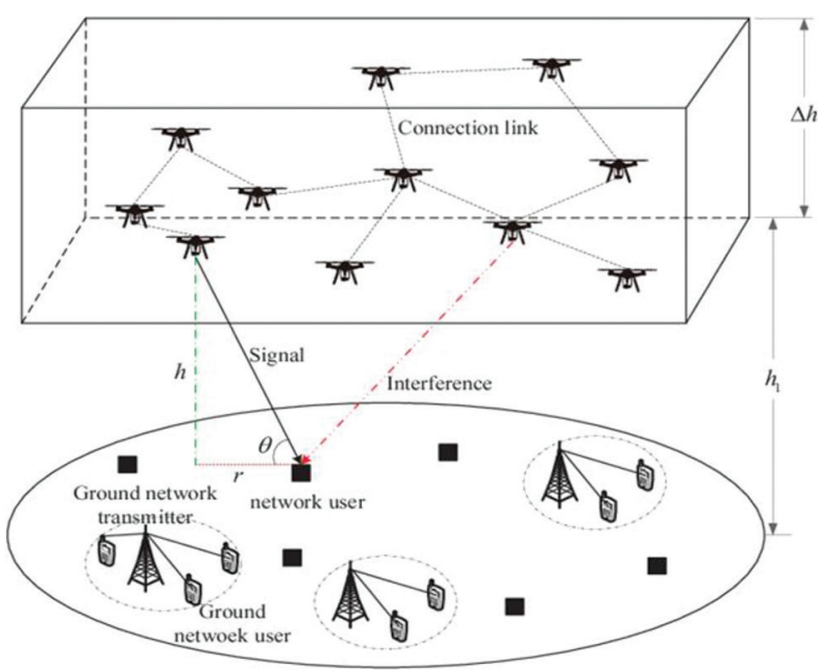

Fig. 1. Flying ad hoc networks [2].

TABLE I

List OF WIFI 802.11N STANDARDS (KarmaKaR, ChatTopadHyAY AND ChaKraborTy, 2017)

\begin{tabular}{llll}
\hline \hline $\begin{array}{l}\text { IEEE } \\
\text { standard }\end{array}$ & $\begin{array}{l}\text { Frequency/ } \\
\text { medium }\end{array}$ & Speed & Transmission range \\
\hline 802.11 & $2.4 \mathrm{GHz} \mathrm{RF}$ & $\begin{array}{l}\text { 1-2 Mbps } \\
\text { Up to } 54 \mathrm{Mbps}\end{array}$ & $\begin{array}{l}20 \text { feet } \\
25-75 \text { feet; range can be } \\
\text { affected by building materials } \\
802.11 \mathrm{a}\end{array} 5 \mathrm{GHz}$ \\
$802.11 \mathrm{~b}$ & $2.4 \mathrm{GHz}$ & Up to $11 \mathrm{Mbps}$ & $\begin{array}{l}\text { Up to } 150 \text { feet; range can be } \\
\text { affected by building materials }\end{array}$ \\
$802.11 \mathrm{~g}$ & $2.4 \mathrm{GHz}$ & Up to $54 \mathrm{Mbps}$ & $\begin{array}{l}\text { Up to } 150 \text { feet; range can be } \\
\text { affected by building materials. }\end{array}$ \\
$802.11 \mathrm{n}$ & $2.4 \mathrm{GHz} / 5 \mathrm{GHz}$ & Up to $600 \mathrm{Mbps}$ & $\begin{array}{l}175+\text { feet; range can be } \\
\text { affected by building materials } \\
175+\text { feet; range can be } \\
\text { affected by building materials }\end{array}$ \\
\hline \hline
\end{tabular}

WIFI: Wireless Fidelity

different video sizes, different type of mobility model, and transmitting with dual bands of IEEE standards by measuring the throughput, retransmission attempts, and delay metrics.

This paper gives extra importance to FANET, as it analyzes different transmission challenges using infrastructure-less FANET flying drones, which is useful for video recording, surveillance, tactical and wireless sensor networks, firefighters, search and rescue teams, and thermal detection which is very important for detecting COVID-19 patients.

The following is the structure of the paper, part two explains the recent related work that analyzed FANET with different scenarios, part three shows and explains the full parameters used for the different scenarios of the paper simulations, part four shows the results and the performance analysis, and finally, part five will conclude the paper with a given future work.

\section{RELATED WORK}

Different researches were published that analyzed and optimized FANET challenges, using several techniques and methods to enhance the performance of data transmission.
In Mahmud and Cho, 2019, a new technique was proposed for low-energy hello interval adaptive signals by choosing the best route to the receiver and minimizing the number of FANET drones used to establish routes. The new technique decreases up to $25 \%$ less from the used energy. In He, Tang, Zhang, Du, Zhou and Guizani, 2019, a new protocol for FANET was proposed course-aware opportunistic routing estimate the best position of the next drone to transmit the data with lower delay and higher throughput, the result shows lower delay with highly performance improvement and better throughput. In Srivastava and Prakash, 2021, a comprehensive survey is presented regarding FANET and discussion about its main critical problems, such as FANET's characteristics, many mobility models, types of possible communication, architecture, categorization, routing protocol, and topologies, also a discussion about FANET's related problems and challenges was analyzed to determine the probabilistic listed points of the research and methods for better results. The researcher in QasMarrogy, 2020, shows an attempt to enhance the video transmission in 5 $\mathrm{GHz}$ frequency in IEEE Wifi standard by changing wireless LAN parameters of MANET nodes, the result shows better performance and throughput with lower delay using specific parameters. Finally, AlKhatieb, Felemban and Naseer, 2020, presented an evaluation and comparison about different types of mobility models such as Pursue Mobility Model, Semi-Random Circular Movement (SCRM), Manhattan Grid Mobility Model (MGM), and Random Waypoint Mobility (RWPM), the paper concludes MGM model effect highly on the packet dropping ratio and delay.

\section{FANeT'S PARAMETER}

In this part, a full explanation will be presented and analyzed regarding the calculated FANET parameters in this paper.

\section{A. Routing Protocols}

Routing protocols are a set of rules that connect drones to guide the transmitted packets from the source node to the destination. There are different types of routing protocols used to connect the flying drones or moving nodes (QasMarrogy, Alqaysi and Almashhadani, 2017), and they can be classified into three types, namely, reactive, proactive, and hybrid routing protocols, as shown in Fig. 2.

In this paper, two types of routing protocols were selected and simulated as they give the highest result for FANET, namely, Ad Hoc On-Demand Vector (AODV) and Optimized Link State Routing (OLSR) Protocol.

Due to the reactive nature of AODV, it broadcast discovery messages tagged with sequence numbers to select a recently updated route to the destination. When the sender sends a packet to the destination the discovery mechanism started, and the sender will record all the fresh recent routes in its routing table to the time that the sender finishes the transmission, and the stored routes will be deleted, then when a new packet will be transmitted another discovery message will be broadcast and the same circle will be repeated, which 
can give more delay to the packets and higher overhead at the beginning of the transmission (QasMarrogy, 2021).

Furthermore, due to the proactive nature of OLSR, it exchanges the final recent information regarding all the latest routes between a group of selected devices or drones called Multipoint Relays, resulting in lower delays during the process of route discovery. After finishing transmission, all the used routes will be stored and used again for the next transmission while collecting more information about more effective routes all the time, this can cause lower delay during transmission but gives higher load as there is too much information broadcasted all the time to prepare available routes continuously (QasMarrogy, 2021).

\section{B. Mobility Models of FANET}

Mobility models show the movement of drones from one location to another location with varying velocity and direction over a specific amount of time. The main challenge in FANET is the varying speed and height of flying drones, which causes the packets to be delayed, failed to be received, or dropped (Chriki, Touati, Snoussi and Kamoun, 2019). Therefore, to break this challenge, the performance of FANET must be evaluated using different mobility models with realistic scenarios that are used in real-life missions. Many researchers use the RWPM to simulate real-life scenarios for FANET, where the drones in this model flies to random directions with random speeds, which lowest the performance as they cause multiple route link breakage between the drones.

In this paper, three different mobility models were simulated, namely, Gauss Markov Mobility (GMM) Model, RWPM, and SCRM Model, as shown in Fig. 3.

The first type of mobility model used in this paper is the RWPM, it uses random times for movements and pauses, and random values for speeds and directions. This model starts when the simulation begins and finishes when the simulation is finished. This randomness in movement and speed will be repeated during simulation time. This model is very important for real-life scenarios such as wireless sensor networks, scanning areas, and search and rescue missions (Sharma and Kim, 2019).

The second type is the SRCM, which uses a route shaped like a hexagon or a circle with a fixed speed to the destination drone, both hexagon area and directions are specified at the beginning of the simulation, and it is used for patrolling surveys and target monitoring (Adya and Sharma, 2020).

The third type is the GMM, it updated the direction and speed of the drone according to their past values during

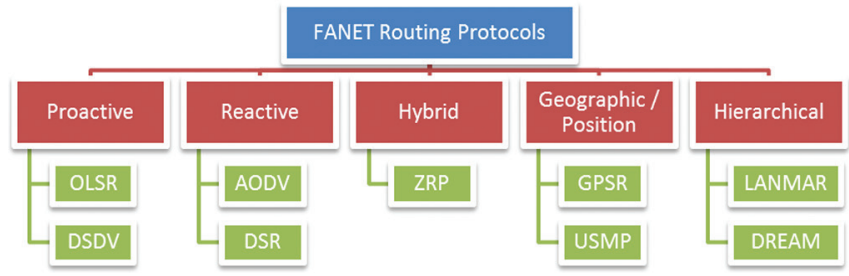

Fig. 2. Flying ad hoc network routing protocols (QasMarrogy, Alqaysi and Almashhadani, 2017). earlier time intervals. Direction and speed calculations are done with a specific degree of randomness that depends on FANET parameters. Finally, each drone movement is independent of all other drones in the same FANET (Korneev, Leonov and Litvinov, 2018).

\section{IEEE WIFI Standards}

In the wireless world, WIFI is a standard means of wireless access transmission, by sending the packet without any cable using radio frequencies, which is a trademark owned by the WIFI Alliance that sets the IEEE of 802.11 wireless standards (Deniau, Gransart, Romero, Simon and Farah, 2017).

One of the standards of WIFI is the IEEE $802.11 \mathrm{n}$ that uses dual B.W frequencies of $2.4 \mathrm{GHz}$ and $5 \mathrm{GHz}$, which considered an essential upgrade to the WIFI standards, by increasing data transmission speed while decreasing the delay and interference (Dolińska, Jakubowski and Masiukiewicz, 2017), Fig. 4 shows a comparison between both $2.4 \mathrm{GHz}$ and $5 \mathrm{GHz}$ in terms of speed, delay, interference, and range.

Data transmission in WIFI can affect the performance of FANET greatly, using $5 \mathrm{GHz}$ frequency can increase the speed of transmission, as it has less network congestion, much reliable WIFI connection, more solid, and lower interference, as it uses more than 20 channel to operate than the $2.4 \mathrm{GHz}$ which uses 13 channels with three non-overlapping channels (1, 6, and 11), as shown in Fig. 5 (Qaddus, 2019). Still, as a disadvantage, it has less coverage range as it has shorter radio waves. Nonetheless, WIFI coverage can be extended using high gain directional antennas (Aziz, Abd Razak and Ghani, 2017).

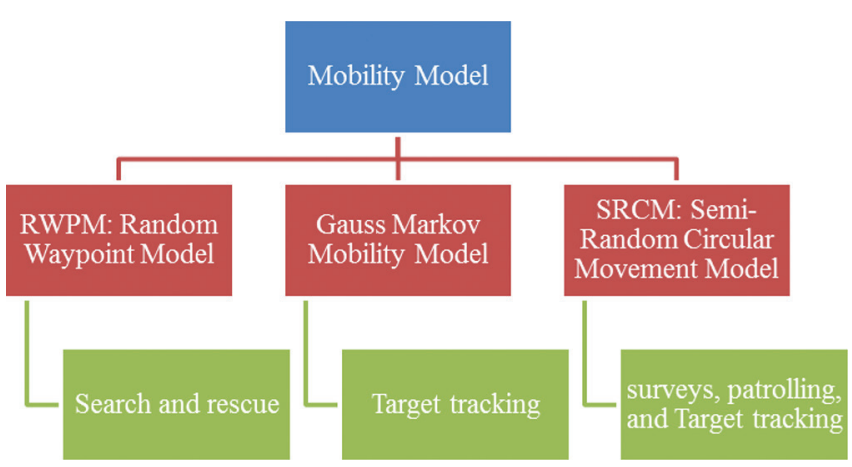

Fig. 3. Real-life mobility model scenarios (Chriki, Touati, Snoussi and Kamoun, 2019).

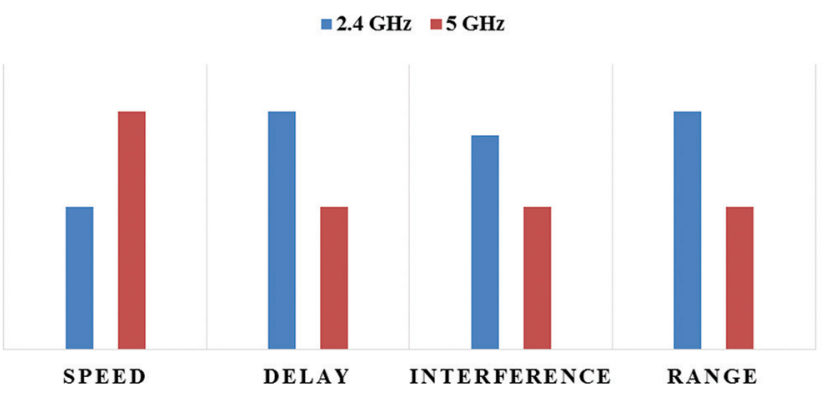

Fig. 4. 2.4 GHz versus $5 \mathrm{GHz}$ comparison (Deniau, Gransart, Romero, Simon and Farah, 2017). 


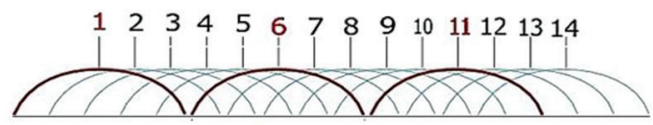

2.4Ghz Channels

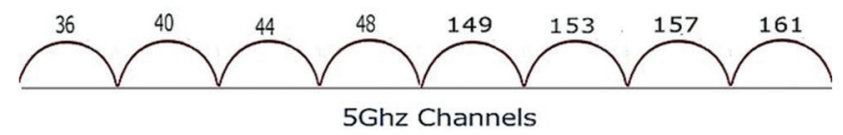

Fig. 5. 2.4 GHz and $5 \mathrm{GHz}$ channel band (Aziz, Abd Razak and Ghani, 2017).

For high-quality video transmission, IEEE 802.11 n $5 \mathrm{GHz}$ frequency will be more required as it can support a higher transfer rate of data as possible (Qaddus, 2019).

\section{RESUlts AND ANALYSIS}

In this paper, a comprehensive analysis of realistic FANET scenarios will be done, to evaluate different aspects of challenges and parameters that facing FANET, to enhance its performance by comparing the results of the simulations together. A $1500 \times$ 1500 square meter area was simulated with 30 drones flying at a height of $20 \mathrm{~m}$, with a varying speed from $1.4 \mathrm{~m} / \mathrm{s}$ (human walking speed) to $20 \mathrm{~m} / \mathrm{s}$ (drone speed), recording two types of video quality, $\mathrm{HD}$ and $2 \mathrm{k}$, moving with three different types of mobility models, namely, RWPM, SRCM, and GMM. These flying drones stream and send the recorded videos from one to one until they reach the destination drone using two types of WIFI IEEE 802.11n standards, $2 \mathrm{GHz}$ and $5 \mathrm{GHz}$, with the support of two types of routing protocols AODV, and OLSR. All simulations were repeated 10 times with average calculations to get the optimal values by calculating the end-to-end delay, throughput, and retransmission attempt of the transmitted packets metrics. Finally, the NS3 simulator was used to simulate FANET as it is one of the best network simulation tools.

To calculate the required B.W for video quality formats, the following equation will be used Equation (1) (Li, Salehi, Bayoumi and Buyya, 2016).

\section{Size of Video $=$ Color Depth $\times$ frame Rate \\ $\times$ Streaming Duration $\times($ Frame $w \times h)$}

To calculate the two sizes of videos chosen in this paper using Equation (1), with size of $1280 \times 720$ pixel for the HD video and $2048 \times 1080$ pixel for the $2 \mathrm{~K}$ video, with a depth color of 240 bits, 30 frame rate, and $60 \mathrm{~s}$ streaming duration. The audio size will be neglected as it does not affect the B.W, the final size will be as follows:

Size of HDVideo $=240 \times 30 \times 60 s \times(1280 \times 720) \approx 47,460 M B$

Size of 2 KVideo $=240 \times 30 \times 60 s \times(2048 \times 1080) \approx 113,906 M B$

The full parameters used in the simulations are shown in Table II.

The results in Fig. 6 demonstrate the streaming of HD video throughput for IEEE $802.11 \mathrm{n}$, routing protocols, and mobility models of FANET. It shows that AODV gives a high
TABLE II

SIMULATION PARAMETERS

\begin{tabular}{ll}
\hline \hline Parameters & Values \\
\hline Video formats & HD $(1280 \times 720)$ pixels, $2 \mathrm{~K}(2048 \times 1080)$ pixels \\
Area size & $1500 \times 1500 \mathrm{~m}^{2}$ \\
FANET routing protocols & AODV, OLSR \\
FANET number & 30 Drone \\
Mobility models & RWPM, SRCM, GMM \\
Simulation time & $1 \mathrm{~min}$ \\
Node speed, height & Varying $1.4-20 \mathrm{~m} / \mathrm{s}, 20 \mathrm{~m}$ \\
WIFI IEEE $802.11 \mathrm{n}$ & $2.4 \mathrm{GHz}, 5 \mathrm{GHz}$ \\
\hline \hline
\end{tabular}

FANET: Flying ad hoc network

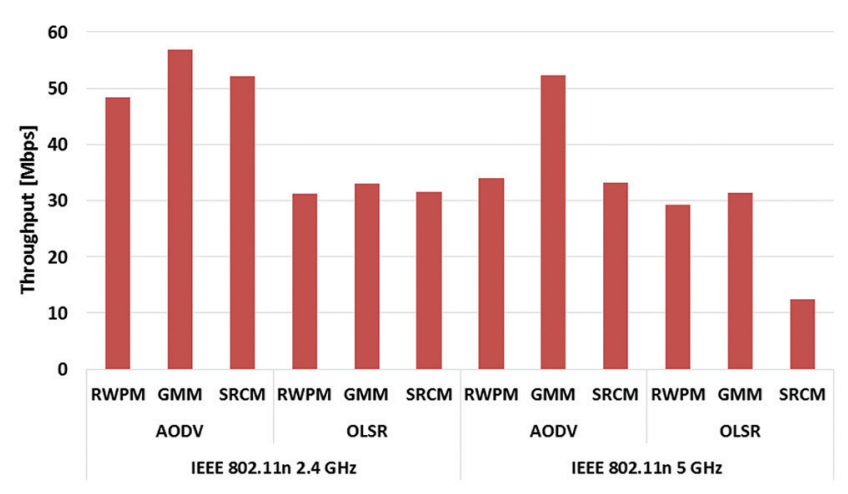

Fig. 6. HD video throughput for IEEE 802.11 n, routing protocols, and mobility models.

result in GMM for the $5 \mathrm{GHz}$ and $2.4 \mathrm{GHz}$ whereas OLSR shows less result. As a reactive protocol AODV requires less B.W to discover a recent fresh route to destination, still it overhead high message at the beginning of the transmission to find recent routes, after that the packets transmitted until the simulation ends. Whereas OLSR keeps transmitting advertising messages to fined routes continuously, but due to FANET movement, the topology is changing all the time which is difficult to keep consistent routes for transmission.

GMM in mobility models shows higher throughput as it keeps the distance and speed from one point to another, this keeps the route fresher for a long time for transmission. RWPM gives the lowest results, as its routes keep changing due to high random mobility direction and speed.

Fig. 7 demonstrates the streaming of $2 \mathrm{~K}$ video throughput for IEEE $802.11 \mathrm{n}$, routing protocols, and mobility models of FANET. It shows that AODV has also higher results as its search for a new route in the beginning of the transmission, therefore, it can keep the route for a longer time. OLSR also shows less result with the RWPM mobility model.

The result in Fig. 8 demonstrates the streaming of HD video delay for IEEE $802.11 \mathrm{n}$, routing protocols, and mobility models of FANET. It shows that the mobility model GMM for AODV gives less delay for both 2.4 and $5 \mathrm{GHz}$, respectively, as AODV uses a fresh route for more time than OLSR, which keeps the route alive for a long time for transmission, therefore, it lowers the delay to find other routes on request.

SRCM and RWPM in mobility models show higher delay as their movement and speed change all the time, which causes the route to be broken. 


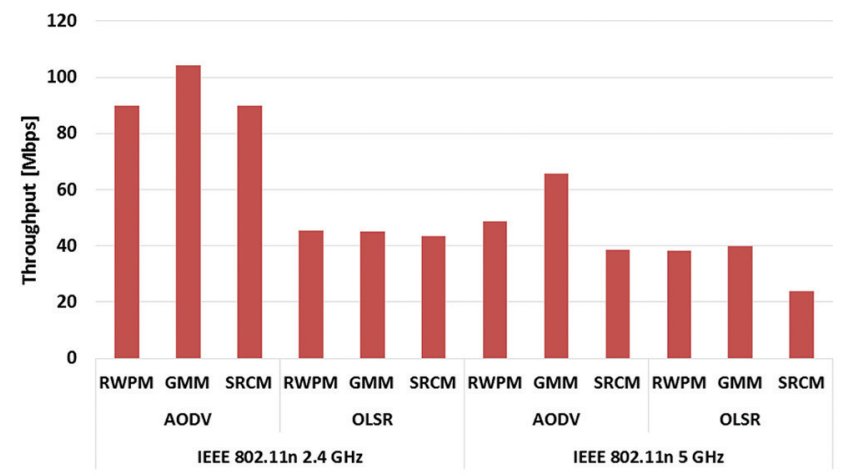

Fig. 7. $2 \mathrm{~K}$ video throughput for IEEE 802.11 n, routing protocols, and mobility models.

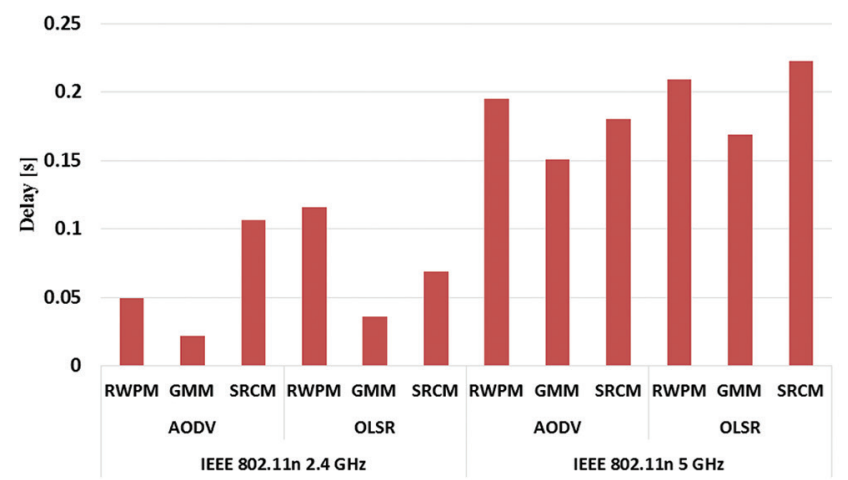

Fig. 8. HD video delay for IEEE 802.11 , routing protocols, and mobility models.

Fig. 9 demonstrates the streaming of $2 \mathrm{~K}$ video delay for IEEE $802.11 \mathrm{n}$, routing protocols, and mobility models of FANET. It shows that $2.4 \mathrm{GHz}$ gives less delay for all mobility models as the distance between the nodes get higher, which is not convenient for $5 \mathrm{GHz}$ as it has small coverage distance, thus causing a higher delay.

The result in Fig. 10 demonstrates the streaming of HD video retransmission attempts for IEEE $802.11 \mathrm{n}$, routing protocols, and mobility models of FANET. It shows that the mobility model GMM for AODV also gives less retransmission attempt for both 2.4 and $5 \mathrm{GHz}$, as the route remains longer than OLSR which support the packet delivery, thus decreasing the retransmission attempts from the drone.

RWPM always shows higher retransmission attempts as the drone movement and speed changes all the time, which causes the route to be broken, and the packet to be dropped and retransmit again.

Fig. 11 demonstrates the streaming of $2 \mathrm{~K}$ video retransmission attempts for IEEE $802.11 \mathrm{n}$, routing protocols, and mobility models of FANET. It shows that $2.4 \mathrm{GHz}$ gives less retransmission attempts for all mobility models as the distance between the nodes get higher, which is not convenient for $5 \mathrm{GHz}$ as it has small coverage distance, thus causing higher route breakage, and more retransmission attempts from the drones.

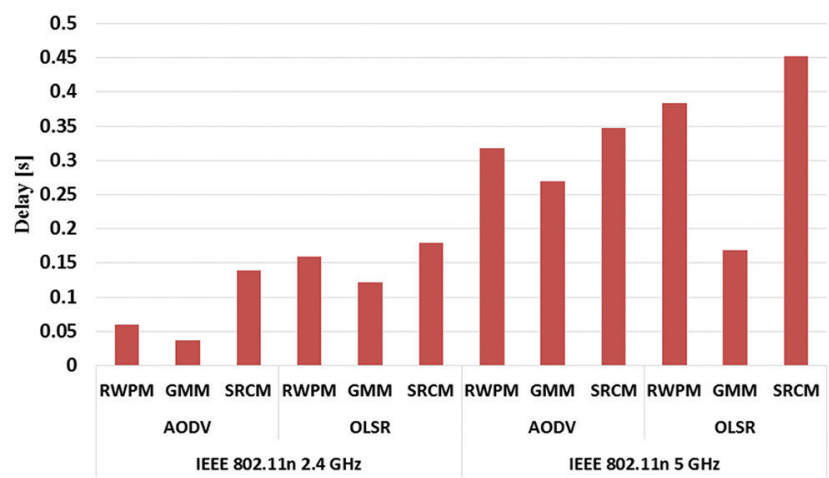

Fig. 9. 2K video delay for IEEE $802.11 \mathrm{n}$, routing protocols, and mobility models.

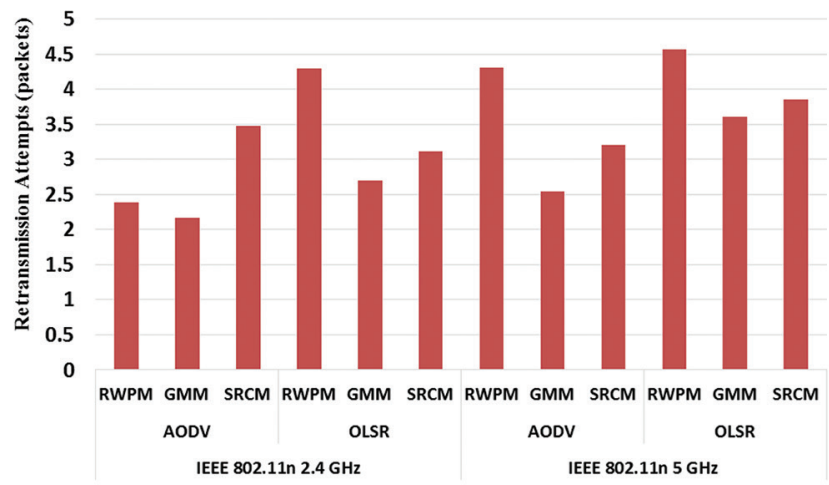

Fig. 10. HD video retransmission attempts for IEEE 802.11 n, routing protocols, and mobility models.

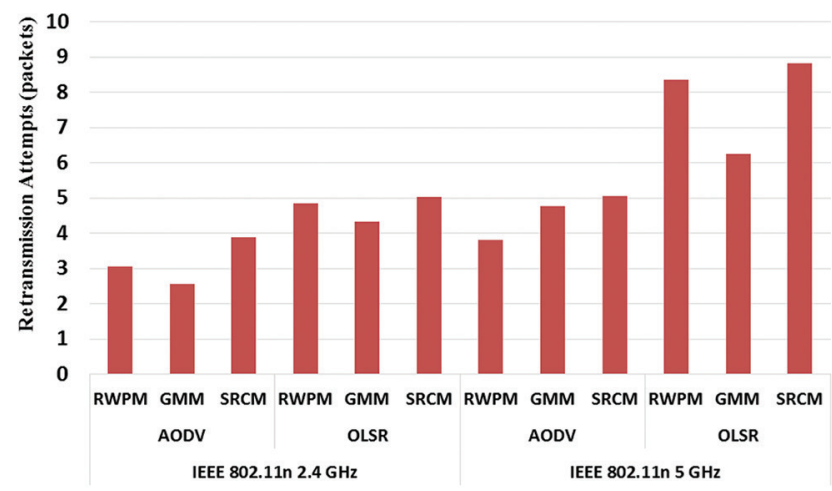

Fig. 11. 2K video retransmission attempts for IEEE 802.11n, routing protocols, and mobility models.

\section{CONCLUSION}

Recently, FANET is considered one of the most important future technologies to be used, as it can achieve more realistic missions without any physical guidance such as thermal detection for COVID-19, search and rescue, military missions, moving objects between different areas, and many more. Still, FANET is facing different types of challenges to achieve better performance for heavy duty missions such as battery life, transmission rate, and coverage, mobility 
models, and more. Thus, it is very important to analyze these challenges of FANET.

In this paper, a comprehensive analysis for FANET was done for 30 drones, flying with varying speed of 1.4, to $20 \mathrm{~m} / \mathrm{s}$, using two types of routing protocol, AODV, and OLSR, with three types of mobility models, RWPM, GMM, and SRCM, while transmitting two types of video format HD and $2 \mathrm{~K}$, using IEEE $802.11 \mathrm{n}$ standards $2.4 \mathrm{GHz}$ and $5 \mathrm{GHz}$ frequencies.

The result shows that the mobility model GMM gives higher throughput and lower delay for both 2.4 and $5 \mathrm{GHz}$ frequencies using the AODV routing protocol, as it keeps the same routes available for a longer time than OLSR, which gives more time for the packets to be transmitted with lower delay, as OLSR keeps changing its routes during topology changes. IEEE $802.11 \mathrm{n} 2.4 \mathrm{GHz}$ gives a better result as it supports more coverage area from $5 \mathrm{GHz}$, therefore. When the node changing its speed and direction randomly, their distance becomes larger, which will be out of the area of coverage for $5 \mathrm{GHz}$ frequency. For mobility models, GMM is better for drones as it keeps its speed and direction with the group, therefore, no route breakage or packet dropping happens.

As FANET is upgrading all the time, future work is needed, to test and simulate more frequencies and more mobility models, with different types of applications and metrics, also different sizes of drones are needed to be analyzed.

\section{REFERENCES}

Adya, A. and Sharma, K.P., 2020. Energy Aware Clustering Based Mobility Model for FANETs. In: Proceedings of ICETIT 2019. Springer, Cham, pp.36-47.

AlKhatieb, A., Felemban, E. and Naseer, A., 2020. Performance evaluation of Ad-Hoc routing protocols in (FANETs). In: 2020 IEEE Wireless Communications and Networking Conference Workshops (WCNCW). IEEE, United States, pp.1-6.

Aziz, T.A.T., Abd Razak, M.R. and Ghani, N.E.A., 2017. The performance of different IEEE802. 11 security protocol standard on $2.4 \mathrm{GHz}$ and $5 \mathrm{GHz}$ WLAN networks. In: 2017 International Conference on Engineering Technology and Technopreneurship (ICE2T). IEEE, United States, pp.1-7.

Chriki, A., Touati, H., Snoussi, H. and Kamoun, F., 2019. FANET: Communication, mobility models and security issues. Computer Networks, 163, p. 106877.

Deng, C., Fang, X., Han, X., Wang, X., Yan, L., He, R., Long, Y. and Guo, Y., 2020. IEEE 802.11 be Wi-Fi 7: New challenges and opportunities. IEEE Communications Surveys and Tutorials, 22(4), pp.2136-2166.

Deniau, V., Gransart, C., Romero, G.L., Simon, E.P. and Farah, J., 2017. IEEE $802.11 \mathrm{n}$ communications in the presence of frequency-sweeping interference signals. IEEE Transactions on Electromagnetic Compatibility, 59(5), pp.1625-1633.

Dolińska, I., Jakubowski, M. and Masiukiewicz, A., 2017. Interference comparison in Wi-Fi $2.4 \mathrm{GHz}$ and $5 \mathrm{GHz}$ bands. In: 2017 International
Conference on Information and Digital Technologies (IDT). IEEE, United States, pp.106-112.

He, Y., Tang, X., Zhang, R., Du, X., Zhou, D. and Guizani, M., 2019. A courseaware opportunistic routing protocol for FANETs. IEEE Access, 7, pp.144303144312.

Karmakar, R., Chattopadhyay, S. and Chakraborty, S., 2017. Impact of IEEE $802.11 \mathrm{n} / \mathrm{ac}$ PHY/MAC high throughput enhancements on transport and application protocols-a survey. IEEE Communications Surveys and Tutorials, 19(4), pp.2050-2091.

Kaur, P. and Singh, A., 2018. Nature-inspired optimization techniques in VANETs and FANETs: A survey. In: Advanced Computational and Communication Paradigms. Springer, Singapore, pp.651-663.

Khan, M.A., Safi, A., Qureshi, I.M. and Khan, I.U., 2017. Flying ad-hoc networks (FANETs): A review of communication architectures, and routing protocols. In: 2017 First International Conference on Latest trends in Electrical Engineering and Computing Technologies (INTELLECT). IEEE, United States, pp.1-9.

Korneev, D.A., Leonov, A.V. and Litvinov, G.A., 2018. Estimation of miniUAVs network parameters for search and rescue operation scenario with Gauss-Markov mobility model. In: 2018 Systems of Signal Synchronization, Generating and Processing in Telecommunications (SYNCHROINFO). IEEE, United States, pp.1-7.

Li, X., Salehi, M.A., Bayoumi, M. and Buyya, R., 2016. CVSS: A cost-efficient and QoS-aware video streaming using cloud services. In: $201616^{\text {th }}$ IEEE/ACM International Symposium on Cluster, Cloud and Grid Computing (CCGrid). IEEE, United States, pp.106-115.

Mahmud, I. and Cho, Y.Z., 2019. Adaptive hello interval in FANET routing protocols for green UAVs. IEEE Access, 7, pp.63004-63015.

Marrogy, G.A.Q., 2020. Enhancing video streaming transmission in $5 \mathrm{GHz}$ fanet drones parameters. Telecommunications and Radio Engineering, 79(11), pp.997-1007.

Qaddus, A., 2019. An Evaluation of $2.4 \mathrm{GHz}$ and $5 \mathrm{GHz}$ ISM radio bands utilization in backhaul IP microwave wireless networks. In: 2019 International Conference on Information Science and Communications Technologies (ICISCT). IEEE, United States, pp.1-5.

QasMarrogy, G.A., 2020. Optimizing video transmission performance in $5 \mathrm{GHz}$ MANET. Journal of Duhok University, 23(2), pp.402-411.

QasMarrogy, G.A., 2021. Improving VoIP transmission for IEEE 802.11 n $5 \mathrm{GHz}$ MANET. Zanco Journal of Pure and Applied Sciences, 33(1), pp.157-162.

QasMarrogy, G.A., Alqaysi, H.J. and Almashhadani, Y.S., 2017. Comprehensive study of hierarchical routing protocols in MANET using simple clustering. In: Conference of Cihan University-Erbil on Communication Engineering and Computer Science, p.62.

Sharma, P.K. and Kim, D.I., 2019. Random 3D mobile UAV networks: Mobility modeling and coverage probability. IEEE Transactions on Wireless Communications, 18(5), pp.2527-2538.

Srivastava, A. and Prakash, J., 2021. Future FANET with application and enabling techniques: Anatomization and sustainability issues. Computer Science Review, 39, p.100359.

Zheng, Z., Sangaiah, A.K. and Wang, T., 2018. Adaptive communication protocols in flying ad hoc network. IEEE Communications Magazine, 56(1), pp.136-142. 